\title{
Solving the Mystery of the Tunguska Explosion
}

\author{
Jian'an Wang \\ Department of Physics, Shenzhen University, Shenzhen, China \\ Email:wja@szu.edu.cn
}

How to cite this paper: Wang, J.A. (2020) Solving the Mystery of the Tunguska Explosion. Journal of Modern Physics, 11, 779-787.

https://doi.org/10.4236/jmp.2020.116050

Received: April 14, 2020

Accepted: May 25, 2020

Published: May 28, 2020

Copyright $\odot 2020$ by author(s) and Scientific Research Publishing Inc. This work is licensed under the Creative Commons Attribution International License (CC BY 4.0).

http://creativecommons.org/licenses/by/4.0/ (c) (i) Open Access

\begin{abstract}
In this paper, the asteroid "rockburst plus nuclear explosion" model of Tunguska explosion is proposed for the first time, and it is believed that the Tunguska explosion is a thermonuclear explosion in the air triggered by a "rockburst" after the asteroid entered the atmosphere, and all the abnormal phenomena produced by the Tunguska explosion are explained by using this model. The "rockburst" is the sudden release of the stress potential energy formed by the expansion of atoms in solid objects caused by the cosmic expansion. According to the "rockburst plus nuclear explosion" model, the "rockburst" was firstly triggered by the heat produced by the friction between the asteroid and the atmosphere after it entered the atmosphere, and then the high temperature and high pressure generated by the "rockburst" ignited the fusion fuel inside the asteroid, resulting in a thermonuclear explosion.
\end{abstract}

\section{Keywords}

Tunguska Explosion, Rockburst, Meteorite Explosion, Thermonuclear Explosion

\section{Introduction}

The Tunguska explosion [1] [2] is an explosion event that occurred at 7:17 a.m. on June 30, 1908, over what is now Siberia's evinci region in Russia. The explosion occurred 800 kilometers northwest of Lake Baikal near the Tunguska river. The estimated explosive power is equivalent to 20 million tons of TNT, or the equivalent of 1000 Hiroshima atomic bombs! More than 80 million trees burned over 2150 square kilometers. In the morning, locals in the northwest of Lake Baikal reportedly observed a huge fireball as bright as the sun streak across the sky, which was illuminated a few minutes later by a bright flash of light. Later, shockwaves from the explosion shattered windows in the vicinity of 650 kilometers, and the mushroom cloud was observed. The explosion was recorded by seismic monitoring sites across Europe and Asia, and the resulting atmospheric 
instability was even detected by the barometrograph, which had just been developed by famous scientists in Britain at the time. In the days after the explosion, the sky over Tunguska, 9000 miles away, was shrouded in a grim orange; the phenomenon of white nights continued in large areas and the night sky was dark red in Asia and Europe. A decrease in atmospheric transparency was also observed at the Smithsonian Astrophysics Station and Mount Wilson Observatory in the United States for at least a few months. At the time of the explosion many lights went out in London, England, and the city was in darkness. The soil in the explosion area was magnetized. Trees in the explosion area grew faster; the width of the ring increased from $0.4-2 \mathrm{~mm}$ to more than $5 \mathrm{~mm}$; genetic variation occurred in some animals; reindeer had a strange skin disease, jujube leper skin disease and so on. No impact crater was found. But Italian nuclear physicists found by heavy isotopes test that the fir trees destroyed in $1908 \mathrm{had}$ higher levels of trace elements than in other years, and those trace elements could not come from earth. There was no sign of a radiation anomaly, indicating that it was not a natural nuclear fission explosion.

A series of unknowns and doubts have given rise to various hypotheses over the past 100 years [3] [4], which can be summarized as the "meteorite impact hypothesis", the "nuclear explosion hypothesis", the "alien spacecraft explosion hypothesis", the "antimatter hypothesis" and the "comet impact hypothesis". Each hypothesis has a certain truth, and each hypothesis can't stand up to scrutiny. So far, no very reasonable explanation has been found.

The purpose of this study is to solve the mystery of the Tungus explosion and to give reasonable explanations for the various abnormal phenomena produced by the Tungus explosion.

\section{The Mechanism of Rock Burst}

\section{1) What is rock burst?}

Rock burst is a phenomenon in which the elastic deformation potential energy accumulated in a rock mass is suddenly and violently released under certain conditions, causing the rock to burst and eject. Rock burst [5] [6] is one of the main safety hazards in deep well mines. A slight rock burst has only flaky rock, no ejection phenomenon, a severe rock burst can produce an earthquake with a magnitude of 4.6 and an intensity of $7-8$ degrees, causing damage to ground buildings and accompanied by loud noises. Rockburst can occur instantly or for days to months.

At present, the scientific community cannot give a reasonable explanation of the source of rock burst energy, which has become the bottleneck of rock burst mechanism research. In the process of studying the geodynamics, the author found that the cosmic expansion is the power source of the earth's geological tectonic movement [7], and further found that rock burst is also a kind of planetary geological tectonic movement, which is a universal natural phenomenon.

2) The relationship between "rock burst" and cosmic expansion 
From Formula (5.31) [8], it can be seen that the orbital radius of the electrons around the nucleus of the atoms or ions that make up the of the object increases with the decrease of the speed of the object moving in the ether. It can be seen that the atoms and ions (ions containing orbital electrons) that make up the objects become larger as the universe expansion slows down or the speed of the objects decreases relative to the cosmic ether system.

The effects of cosmic expansion on planets composed of solid crust (lithosphere) and liquid magma are very rich, including orogeny, epeirogenic movement, plate formation, earth expansion, sea floor spreading, continental drift, earthquake and volcanic eruption [7]. If we focus on the effect of cosmic expansion on solid objects, it is not difficult to find that the so-called "rock burst" is the sudden release of elastic potential energy formed by the long-term influence of cosmic expansion on solid objects. This release of elastic potential energy can be either spontaneous or artificially induced.

We can approximate the shape of the solid to the sphere for easy study. If the surface of a solid sphere remains unbroken (keeping the atoms next to each other), the surface area of the solid sphere is proportional to the square of the radius of the atoms forming the sphere, and the volume of the solid sphere is proportional to the cube of the atomic radius. So, as the atoms expand, the volume of the solid ball increases faster than the surface area increases, the stress in the solid ball gets bigger and bigger, and the deeper in the ball, the bigger the stress.

Since some naturally forming solid objects in the universe, such as meteors or asteroids, have been formed for billions or even over 10 billion of years, the stress that accumulates in these solid objects after billions or 10 billion of years of expansion in the universe is very large and the solid object will explode when the stress is greater than the structural force of the solid object. This is why there are so many irregularly shaped meteors (meteorites) in the universe. Because the small planets completely solidified so early, as the universe expanded, the stresses inside the solid planets outweighed their structural forces and exploded (rock burst), resulting in the formation of large numbers of irregularly shaped rocks (meteorites or meteors). So we can predict that if the universe continues to expand, the moon and all the planets will eventually become solid and explode into rubble (this could be the last violent scene of the tectonic movement).

As the atoms expand, the expanding force inside the rock increases and so does the surface tension of the rock. Once the surface tension exceeds the structural force, the surface of the rock will crack into powder or brittle pieces and fall off layer by layer. This is the process of desert formation.

It's not hard to explain why some meteorites explode when they enter the atmosphere. Because of the extremely fast running speed, when the meteorite falls into the atmosphere, it is heated by intense friction with the atmosphere, and when the temperature of the meteorite reaches the temperature that can release the stress potential energy inside the meteorite, these stress potential energy will be released in the form of explosion. Therefore, all naturally formed solid objects 
in the universe contain stress internal energy, and the older a solid is formed, the higher the stress internal energy it contains. All the Earth's crustal rocks contain stress, the longer the rock formation, the higher the stress. These stresses will not be released when the rock bed is not destroyed because of the protection of the surrounding rock mass, and will suddenly be released when the surrounding rock mass is destroyed (such as tunnel construction), which is the mechanism of the artificial "rock burst".

\section{The "Rock Burst plus Nuclear Explosion" Model of the Tunguska Explosion}

Because of the enormous energy released by the Tunguska explosion (equivalent to 20 million tons TNT explosive, or 1000 Hiroshima nuclear bombs), the authors suspect that the Tunguska explosion is a thermonuclear explosion triggered by a "rock burst" triggered by the asteroid's entry into the atmosphere.

1) The "Rock burst plus nuclear explosion" model of the Tunguska explosion

a) The principle and structure of the hydrogen bomb:

A hydrogen bomb is a nuclear reactor that uses the nuclear fusion of hydrogen isotopes (deuterium and tritium) to release a large amount of energy instantly. Since light nuclei that produce fusion reactions all carry positive charges, a significant fusion reaction can occur only when they move very fast to overcome the electrostatic repulsion between positive charges. If the temperature of the thermonuclear loading is very high (only at temperatures above 14 million degrees ${ }^{\circ} \mathrm{C}$, the reaction speed is large enough to achieve self-sustaining fusion reaction), the nuclei that make up the loading have a very high velocity (and thus a high kinetic energy). The fusion reaction that takes place using this method is called thermonuclear fusion reaction, or thermonuclear reaction. Because the hydrogen isotopes deuterium and tritium in light nuclei have the least repulsion between nuclei. Therefore, it is often chosen as the charge for hydrogen bombs. The main reaction mode between deuterium and tritium nuclei is as follows:

$$
\mathrm{D}+\mathrm{T} \rightarrow 4 \mathrm{He}+\mathrm{n}+17.6 \mathrm{MeT}
$$

where, $D, T, 4 \mathrm{He}$ and $\mathrm{n}$ represent the deuteron, tritium, helium and neutron respectively.

The general structure of the hydrogen bomb is shown in Figure 1. The shell of the hydrogen bomb contains deuterium and tritium or solid lithium deuteride 6 (the nuclear material of hydrogen bombs), multiple atomic bombs, and detonators made from ordinary explosives. The hydrogen bomb uses the high temperature and high pressure generated during the explosion of the atomic bombs to ignite its thermonuclear material for nuclear fusion reaction (or thermonuclear reaction). When the detonator causes a conventional explosive to explode, it quickly compacts the separate blocks of nuclear material, creating fission reactions known as the atomic bomb explosion. The explosion of the atomic bombs immediately produced the ultra-high temperature and pressure needed to fuse 


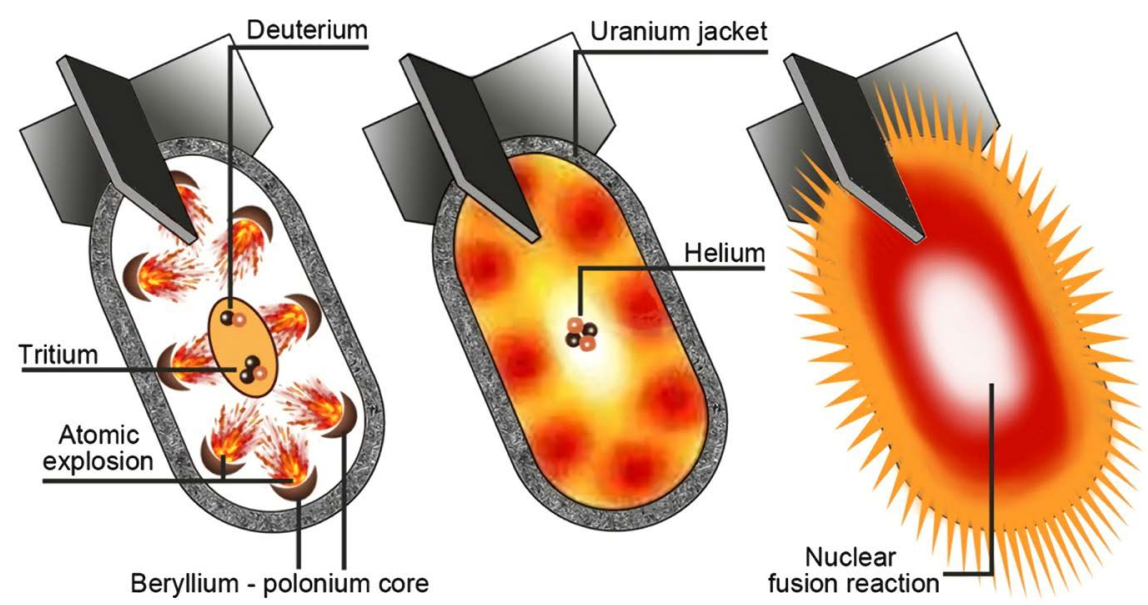

Figure 1. The principle and structure of the hydrogen bomb.

deuterium and tritium. Deuterium and tritium collide with each other at a speed of several hundred kilometers per second, and react quickly and violently to synthesize helium, releasing a lot of fusion energy, thus completing the whole explosion process of the hydrogen bomb. Since thermonuclear material loads have no critical mass limit, the hydrogen bomb can be made very large, with the average explosive power ranging from hundreds of thousands of tons to millions of tons, or even tens of millions of tons of TNT equivalent.

b) How does an asteroid produce a nuclear explosion?

The author suspects that the Tunguska explosion is a nuclear explosion in the air caused by an asteroid formed billions or ten billion of years ago with enough solid deuterium and tritium in its center, as shown in Figure 2. When the asteroid enters the atmosphere, it heats up because of friction with the atmosphere, which leads to the sudden release of the stress potential energy gathered inside the asteroid, or the occurrence of rock burst. The rock burst causes the pressure and temperature at the center of the asteroid to rise dramatically, causing deuterium and tritium at the center of the asteroid to explode in thermonuclear fusion reactions. The process of the asteroid entering the atmosphere is equivalent to the detonation of the atomic bomb in the hydrogen bomb, and the rock burst of the asteroid is equivalent to the explosion of the atomic bomb in the hydrogen bomb. Both ended up with deuterium and tritium fusing at high temperature and high pressure to produce a nuclear explosion. The debris created after the asteroid hydrogen bomb exploded continues to produce rockburst until it is completely turned into charged dust.

Since all elements with a smaller atomic weight than iron can release energy in fusion reactions at high temperatures and pressures, the possibility that the asteroid's nuclear explosion was caused by fusion reactions of other elements, except deuterium and tritium, cannot be ruled out.

Because hydrogen is the most abundant element in the universe and accounts for about $75 \%$ of its mass, asteroids or meteorites containing deuterium and tritium should be ubiquitous in the universe. 


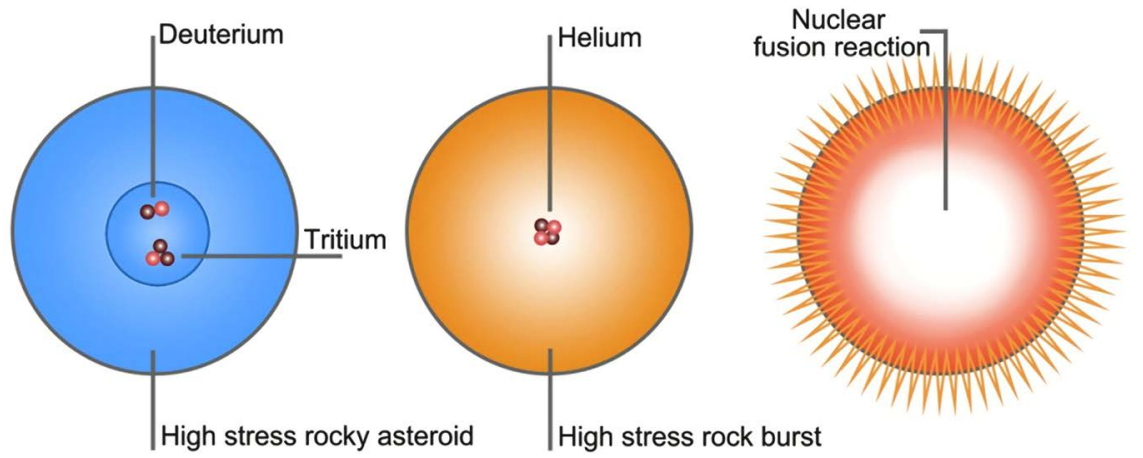

Figure 2. How does an asteroid produce a nuclear explosion?

A high-stress asteroid that is massive enough, and old enough to form, can produce a "rockburst" with the power comparable to that of an atomic bomb, so it can detonate a thermonuclear explosion.

2) Using the model of "rock burst plus nuclear explosion" of the Tungus explosion to explain the abnormal phenomena produced by the Tungus explosion

a) The energy released is huge: the estimated explosive power is equivalent to 20 million tons TNT explosive, equivalent to 1000 Hiroshima atomic bombs!

Answer: since there is no critical mass limit on the amount of thermonuclear material a thermonuclear bomb can contain, an asteroid thermonuclear bomb can be very large, with an explosive yield of tens of millions of tons of TNT or more.

b) The sky was unusual: the 9000-mile-wide sky of the Tunguska region was shrouded in a gloomy orange color in the days following the explosion, with white nights appearing in large areas and dark red in the skies of Asia and Europe. A decrease in atmospheric transparency was also observed at the Smithsonian Astrophysical Station in the United States and the Mount Wilson Observatory for at least a few months.

Answer: because the asteroid was blasted into the atmosphere as electrically charged dust, the sky over the explosion area was covered in this dark orange color. Because the dust that pervades the atmosphere reflects the sunlight, the white night phenomenon appeared, and because the red light is the most penetrating, the night sky appeared dark red. Because the dust in the air is very small so it falls slowly, and because the dust fills a lot of space and reaches a high altitude, it takes a long time for the dust to spread out, so the transparency of the atmosphere was reduced for several months.

c) At the time of the explosion, many lights in London, England, suddenly went out and the city was dark; the soil in the explosion area was magnetized; trees in the explosion area grew faster; ring width increased from $0.4-2 \mathrm{~mm}$ to more than $5 \mathrm{~mm}$; some animals developed genetic mutations, and reindeer developed a strange skin disease, such as date toadiness.

Answer: the asteroid was blasted into electrically charged dust, which, along 
with the rapid fluctuations of the blast wave, produced a powerful electromagnetic pulse that caused the electrical system in London, England, to trip, causing many lights to suddenly go out. It is this electromagnetic pulse, or the high-energy microwaves in it, that have caused the magnetization of the soil in the explosive area, the accelerated growth of the trees (the width of the rings has increased from $0.4-2 \mathrm{~mm}$ to more than $5 \mathrm{~mm}$ ), and genetic variations in certain animals (e.g. strange skin disease of reindeer, jujube leper, etc.)

d) No impact crater was found; but using heavy isotopes, Italian nuclear physicists measured that the amount of trace elements of fir destroyed in 1908 is higher than in other years, and these trace elements can't come from earth, obviously, the explosion is related to meteorites. Survey teams in the 1950s and 1960s found tiny glass balls sprinkled on the ground in the area. Chemical analysis showed that the spheres contained large amounts of nickel and iridium-metals commonly found in meteorites-and determined that they came from beyond earth.

Answer: because each point of the asteroid contains stress, under the heat of the main "rock burst" and "nuclear explosion", the asteroid was blown into dust through a series of secondary" rock burst", so there was no crater left. Because asteroids contain these trace elements, dust containing these trace elements at the time of the big explosion entered the destroyed fir under the action of the explosion shock wave. In the blast zone, the heat melted particles of glass in the soil, mixing them with the dust particles that formed in the explosion. After the explosion, the melted glass particles cooled and solidified to form tiny glass spheres containing nickel and iridium, the metallic elements commonly found in meteorites.

e) There was no sign of a radiation anomaly, indicating that it was not a natural nuclear fission explosion.

Answer: Because the nuclear fusion reaction in the asteroid is caused by "rock burst", not by atomic bomb explosion, there is no nuclear fuel (radioactive element) that produces nuclear fission reaction, so there is no radioactive pollution.

\section{Conclusions}

1) The Tunguska explosion may have been a thermonuclear explosion in the air after the asteroid entered the atmosphere, and the nuclear explosion may have been triggered by the high temperature and high pressure caused by a rock burst of the asteroid; the rock burst is triggered by the high temperature caused by the friction between the asteroid and the atmosphere.

2) Since all elements with less atomic weight than iron can release energy in fusion reactions at high temperatures and pressures; the asteroid's nuclear explosion may have been caused by fusion reactions involving elements other than deuterium and tritium.

3) "Rockburst" is a physical phenomenon of sudden release of the stress potential energy formed in a solid body under the long-term action of cosmic expansion. "Rock burst" is the inevitable (final) process in the evolution of solid 
planets.

4) As the universe expands, the atoms and ions (ions containing orbital electrons) of everything in the universe keep getting bigger. Since the surface area of a solid is proportional to the square of the radius of the atoms that make up the solid, and the volume of the solid is proportional to the cubic of the radius of the atoms that make up the solid, the stress in the solid becomes larger as the atom expands, and the "rock burst" occurs when the stress in the solid is greater than the sum of the structural force of the solid and the external environmental pressure.

\section{The Main Contributions and Key Novelties in the Paper}

1) This paper proposes for the first time that the Tunguska Explosion is a thermonuclear explosion caused by the high temperature and high pressure generated by the asteroid's "rock burst".

2) In this paper, it is pointed out for the first time that "rockburst" is a physical phenomenon of sudden release of the stress potential energy formed in a solid body under the long-term action of cosmic expansion, and that "rockburst" is the inevitable (final) process in the evolution of solid planets.

3) This paper presents for the first time the mechanism of "rockburst" formation: As the universe expands, the atoms and ions (ions containing orbital electrons) of everything in the universe keep getting bigger. Since the surface area of a solid is proportional to the square of the radius of the atoms that make up the solid, and the volume of the solid is proportional to the cubic of the radius of the atoms that make up the solid, the stress in the solid becomes larger as the atom expands, and the "rock burst" occurs when the stress in the solid is greater than the sum of the structural force of the solid and the external environmental pressure.

\section{Future Works}

1) Because any naturally formed solid object contains stress, and the older the solid object is formed, the greater the stress it contains, it is possible to see if an explosion will occur and measure the energy generated by the explosion by heating natural diamond or other relatively old solids, thereby experimentally verifying that the stress energy of "rock burst" is formed by the expansion of atoms caused by the expansion of the universe.

2) Because asteroids like or more powerful than the asteroid that caused the Tunguska explosion should be widespread in the universe, monitoring asteroids that could meet the earth and detonating them artificially by laser or other means is an important task for humans to protect the earth from devastating disasters like the Tunguska explosion.

\section{Conflicts of Interest}

The author declares no conflicts of interest regarding the publication of this paper. 


\section{References}

[1] Chyba, C.F., Thomas, P.J. and Zahnle, K.J. (1993) Nature, 361, 40-44. https://doi.org/10.1038/361040a0

[2] Xu, D.Y. (1986) Journal of Catastrophology, No. 1, 115-117.

[3] Yi, M. (2011) Science \& Technology Vision, No. 15, 25.

[4] Perkins, S. (2008) Science News, 173, 5-6. https://doi.org/10.1002/scin.2008.5591731904

[5] Ortlepp, W.D. and Stacey, T.R. (1994) Tunnelling and Underground Space Technology, 9, 59-65. https://doi.org/10.1016/0886-7798(94)90010-8

[6] Zhang, J. and Fu, B. (2008) Chinese Journal of Rock Mechanics and Engineering, 27, 2034-2042.

[7] Wang, J.A. (2020) Journal of Modern Physics, 11, 407-431. https://doi.org/10.4236/jmp.2020.113026

[8] Wang, J.A. (2019) Journal of Modern Physics, 10, 1615-1644. https://doi.org/10.4236/jmp.2019.1014107 